\title{
Research of Resource Model for Mobile-Learning Based on Cloud Computer
}

\author{
Li donghui $^{\text {a }}$, Dai xiaopeng ${ }^{\mathrm{b}}$ \\ School of information Science \& Technology of Hunan Agricultural University ChangSha China \\ adoner@263.net, 'baixpedu@126.com
}

Keywords: Resource model;Moblie-Learning;Cloud computer.

Abstract: In mobile learning concept . In the first part, the reconstruction of the digital learning resources classification model is established, and then the the cloud based education platform "teacher student subject knowledge ontology" three-dimensional space application model based on. Finally, it designs the subject knowledge ontology resource information model, fully embodies the learning resources evolution, distribution and openness, to achieve basic education learning resources of convergent evolution and semantic polymerization, small granularity reuse and sharing, provides a good protection,

\section{Introduction}

With the wide application of smart phones, tablet PCs and other mobile terminals, has brought a revolution in learning, using lifelong learning become possible.E-learning is growing very fast and many Universities and companies are already supporting in some way an e-learning solution. The rush in the field of wireless and mobile technologies creates opportunity for new field of research - so called 'mobile learning'.Mobile learning has become an important way for people to acquire knowledge, construct knowledge and apply knowledge.Changed the traditional learning mode, and provides a powerful support tool for lifelong learning, universal learning and personalized learning. However, the core of mobile learning is the construction of mobile learning resources. In this paper, based on the characteristics of mobile learning, this paper analyzes the related technologies of the construction of mobile learning resources, and puts forward a kind of mobile learning resource model based on cloud computing.

\section{Mobile learning and mobile learning resources}

\section{Meaning of mobile learning}

M-learning ${ }^{[1]}$ is often defined as e-learning through mobile computational devices. In general by mobile device we mean PDAs and digital cell phone, but more generally we might think of any device that is small,autonomous and unobtrusive enough to accompany us in every moment in our every-day life, and that can be used for some form of learning. These small tools can be seen as instruments for accessing content, either stored locally on the device or reachable through interconnection. They can also be a tool for interacting with people, via voice and through the exchange of written messages, still and moving images.

There are many properties that differ when comparing a mobile device from a desktop PC (the usual medium to deliver e-learning) and they have impact on what is reasonable, useful and even pleasant to do on such devices. Some of them are the output (i.e. the screen size and resolution capabilities, etc.); input (i.e.keypad, touch-screen, voice input); processing power and memory; supported applications and media types. When we try to transfer services provided by an e-learning platform into services in an m-learning platform we can see that some of them should change to fulfill the limitations of the small devices, some are impossible to be delivered in a certain context, but also new services appear, rovoked by the mobility. 
The Connectivity-Contrary to e-learning, which supposes always-on connection, m-learning could be delivered in three different ways. We can schematically call them "pure connection", "pure mobility" and mixture of the previous two (intermitted connection). "Pure connection" is when the mobile device is always connected to internet. Now there are quite a lot of technological ways of having the WWW and other services available for the small devices, i.e. through WAP, GPRS, UMTS, Bluetooth, etc. On the other hand "pure mobility" is when no connection is available and so all the data the applications need should be uploaded on the device and used offline. In this case nowadays mobile phones, which still have very limited memory, can not be used. But the situation quickly changes and the new generation cell phones have more processing power, memory and embedded software. The PDA's could be used now but they also have memory limitations that should be considered though they can be evaded by using extension packs with extra memory. If so delivering also sound/video-lectures offline could be possible.

The Devices' hardware/software characteristics-Access to the web through personal electronic devices, with their small screen size, has been an interesting problem for lots of researchers. Unfortunately, todaymost web pages are designed to be displayed on desktop computers with color monitors having at least 800x600 resolution. This leads to at least 2-to-1 (often greater) ratio of designed vs. available screen area,making direct presentation of most pages on the small devices aesthetically unpleasant, un-navigable, and in the worst case, completely illegible. Depending on the devices used the delivery format and the needed transformations on it could defer. In some cases if we think about WAP devices some transcoding techniques could be used to transform from one presentation language to another (WAP-HTML-WAP). Although it is possible to deliver content to WAP phones the reading is rarely easy enough and the interaction is quite a difficult task. One can also think of delivering the content in alternative ways (e.g. voice, video). When we consider PDAs instead of WAP devices the possibilities are wider, but still all problems linked with conversion/adaptation/transcodification of general purpose content remain.

The New Context (Location-awareness)-The mobility of the devices used in m-learning scenarios involves a new context data to be considered - location. Services involving location-discovery are for example a student/teacher receiving directions how to get to a certain room or alerts for seminars/lectures that can be triggered while taking into consideration the current place and the time to get to the needed aula, location aware printing of the learning content, etc.

\section{The life cycle of mobile learning resources}

The core of mobile learning is the construction of mobile learning resources, and the life cycle of mobile learning resources is divided into four stages: ${ }^{[3]}$ the construction stage, the selection stage, the organization stage and the service stage. As figure 1 shows: construction phase to achieve the reconstruction of digital learning resources of mobile learning resources; selection phase is the construction of learning resources for evaluation, selection and sorting, ensure the quality of learning resources; organization stage is according to the behavior and characteristics of learners, learning the semantics of resources construction, personalized knowledge recommendation service; service stage with mobile learning platform for the realization of learning resource personalized knowledge recommendation service. 


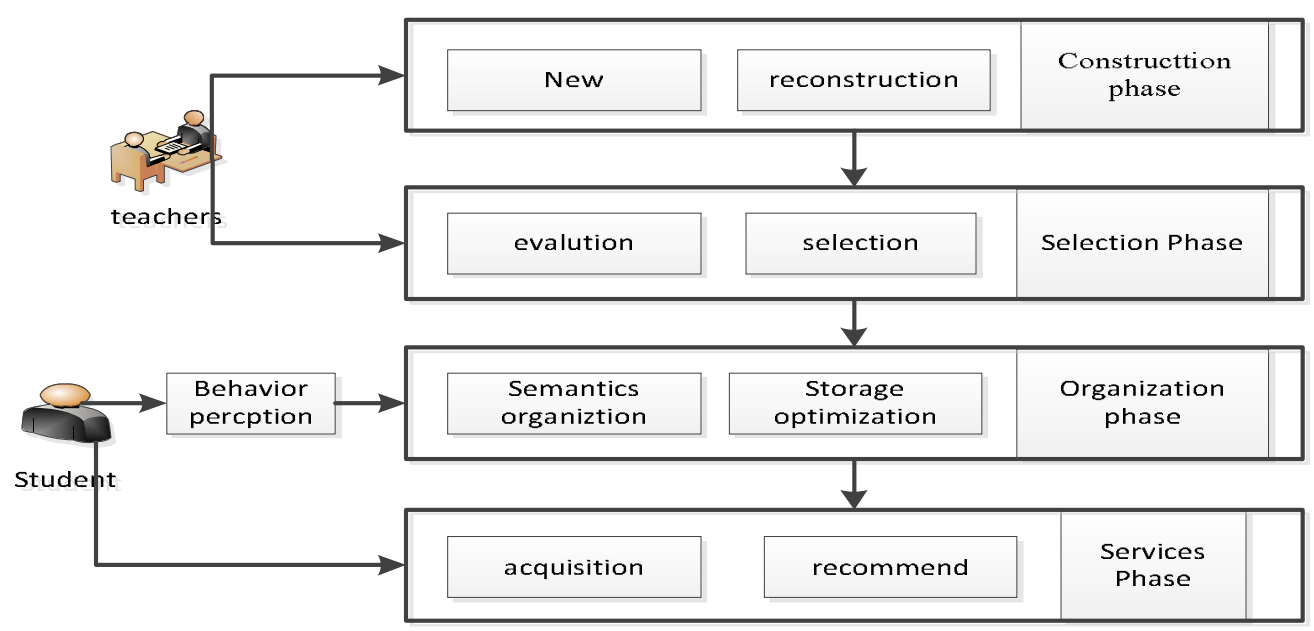

Figure 1 the life cycle of mobile learning resources

\section{M-Learning resource classification model}

We think in the basic education cloud, the basic education resource is a dynamic network distributed storage structure model ${ }^{[4]}$, As shown in Figure 2, it can be divided into subject knowledge ontology resources, process oriented information Sources, social cognitive network resources, teaching resources and learning resources, five major categories.etc

In this model, the dynamic, evolutionary, complexity, openness and situation of learning resources are fully reflected. Among them, the process resource is mainly generated by the individual and social cognitive network and knowledge resource ontology interaction in the process of resources, including information generation, collaborative editing tools and activities, evaluation records, records, and virtual learning partner, teaching and learning situation creation tools, API interface information, survival period and local cloud storage physical address information, semantic description information etc.. Process information is stored in the local cloud server. And not learning resource metadata - "meta learning" are part of the structure, fully embodies the characteristics of process resource life cycle, social context, open-ended evolution, quality control. Meta learning or metadata is building a subject knowledge resource ontology is the smallest unit, no longer attached to process information, only in the body of knowledge resources and knowledge of social cognitive network interaction will produce, adequate protection of the "meta learning" reuse or splitting of the particle size. 


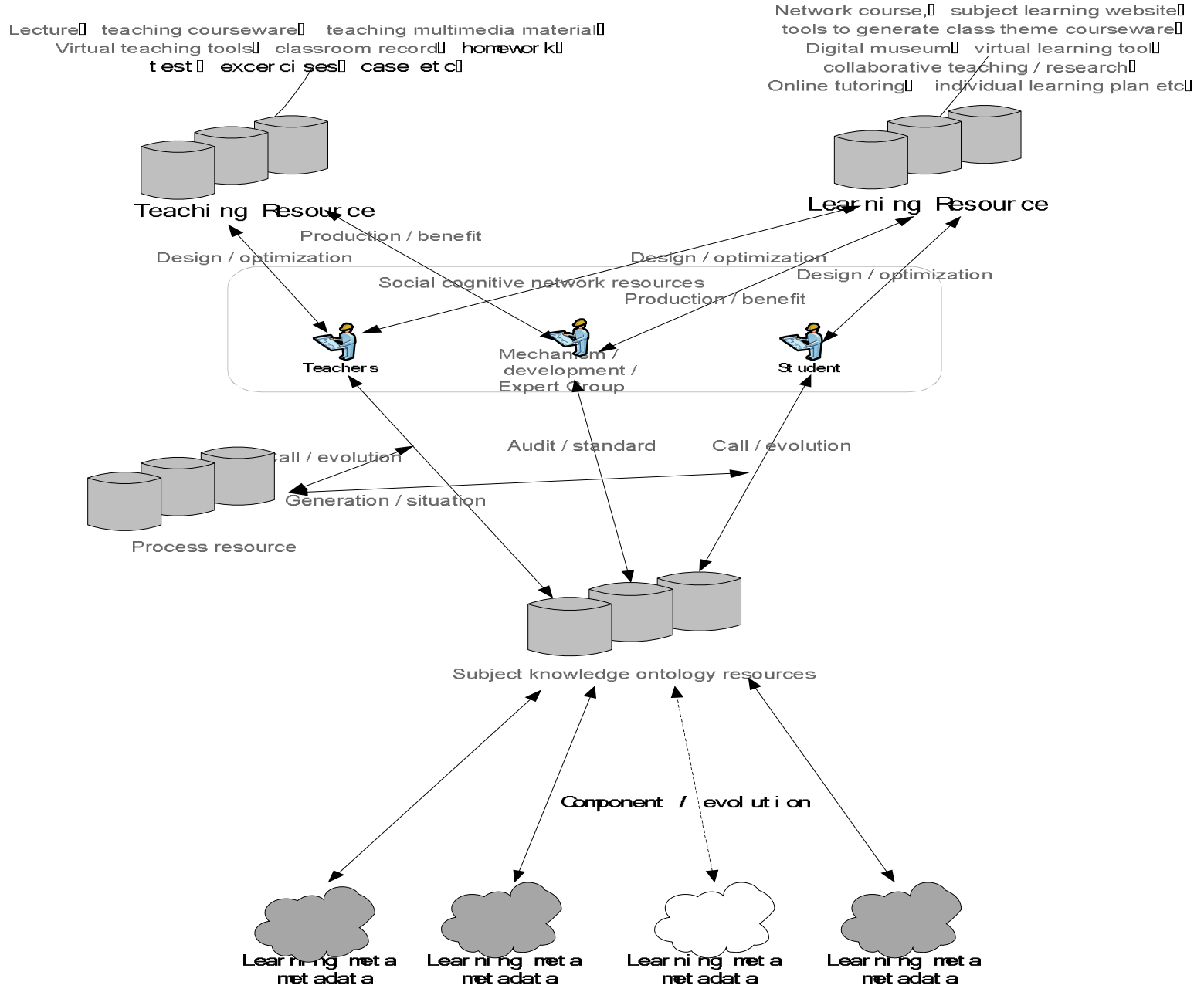

Figure 2 Digital resource classification model based on education cloud

\section{M-Learning resources space model}

Cloud space application of digital learning resources mainly consists of four elements: Education cloud platform, subject knowledge ontology of space resources, teacher and student space form in education under the cloud platform three-dimensional space model (as shown in Figure 3).

Generally speaking, the structure of educational cloud consists of four layers. The upper layer of the education cloud is the learning resource layer (ERaaS) and the educational platform layer (EPaaS). The lower is the universal platform layer (PAAS) and infrastructure (IAAs), and based on the classification of educational resources, the education resources layer ERaaS includes education data service layer EDaaS and education software application service layer (the core of EASaaS) cloud based education application is learning resource service, establish service standards, open the service fields, architecture learning resources group to build a shared services model and structure of the system, is the key for the future work in basic education information. 


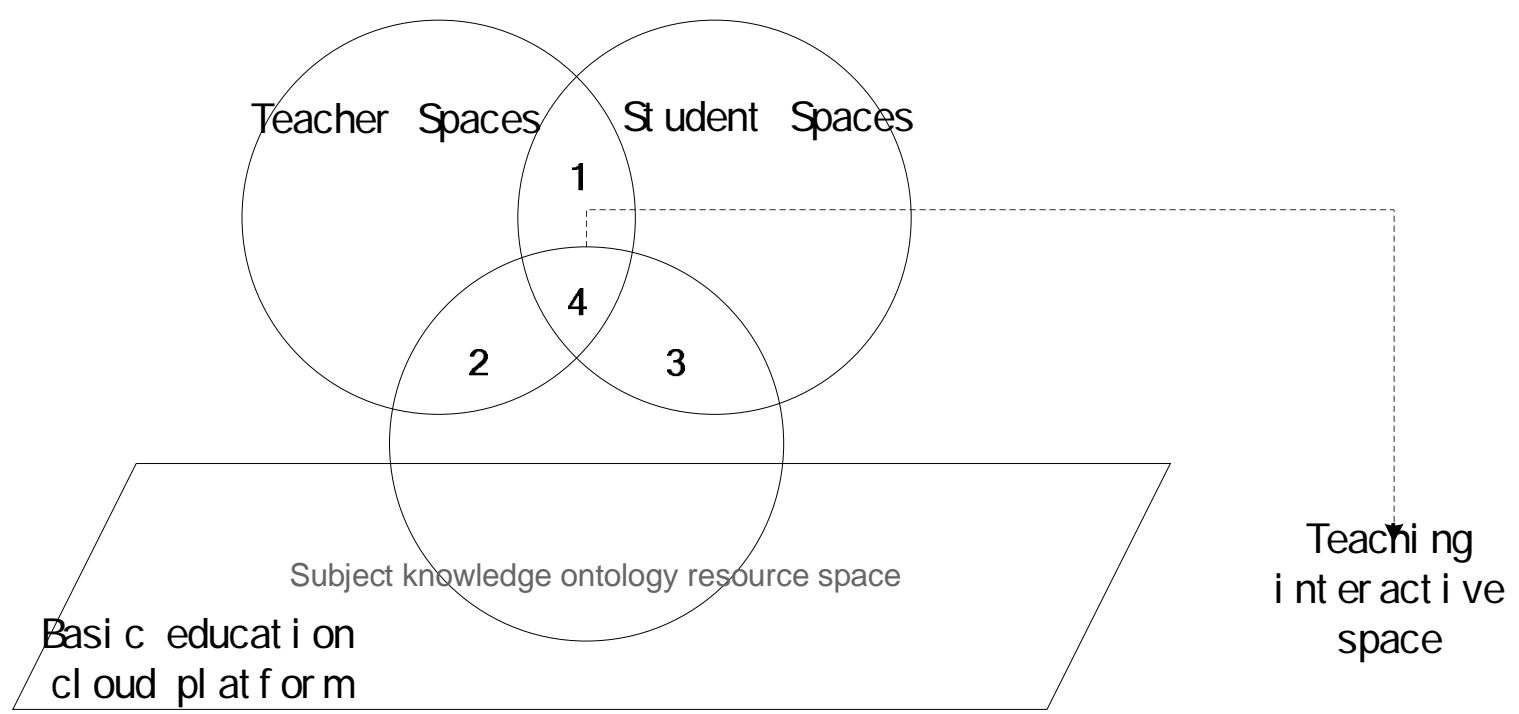

1-Teacher-st udent rel at $i$ onshi $p$ net work 2-Teacher cogni ti ve net work 3-Student cogni ti ve net work 4-Teaching interact i ve space

Figure 3 Application space model of digital learning resources based on education cloud

\section{The service mode of learning resources and the structure of distributed storage}

Basic education cloud, which is the application of cloud computing technology and related services ${ }^{[5]}$ in the field of basic education, is the future development of basic education information technology. It contains all the necessary software and hardware computing resources, and provides a good platform for the construction of future digital learning. The use of open architecture, open source, open standards, open interface, cloud based education to educational institutions, teachers, students, researchers provides intelligent terminal good learning resource integrated service based on pervasive computing.

\section{Teacher space and student space}

In the digital age, learning is no longer a person's activities, develop, and maintain a variety of information links is the assurance of continuous learning, optimizing internal and external network learning process; learners party to construct the internal cognitive structure. On the other side and to construct external social cognitive network - know from which people can acquire certain party and the knowledge and wisdom, and build a sustained and stable social interaction relationship. Therefore, in the space of teachers and students, mainly provide is social cognition sharing network.

\section{Subject knowledge ontology resource space}

With development of Web3.0, pervasive computing, cloud computing technology, learning resources gradually to cloud services run in smart phone, pad, book bag, interactive electronic whiteboard cloud personal learning environment and equipment, original single point of storage of massive resources, in be reused in the clouds the closed, static, not self adapt to problems, serious impact on the cloud service system in the sharing and interoperation, highlights the education resource description model scalability problems. Ontology (Ontology), as the core technology of semantic Web, is the best way to describe the concept hierarchy and semantic model, and it is an effective method to solve the knowledge interaction, sharing and reusing. Mo therefore, ontology and semantic description become one of the core technologies to understand and solve the massive aggregation of learning resources in the cloud platform. So called discipline knowledge ontology resources space, it refers to 
the use of ontology technology and semantic description of the standardization and formalization of the subject knowledge structure of the organization of knowledge ontology space.

\section{Acknowledgments.}

Project supported by the ResearchScience \& Technology Bureau of Hunan Province,China(Grant No. 2014FJ6046).

\section{References}

[1] $\mathrm{Yu}$ Shengquan.From knowledge transmission to congnition constructure, then to contextual cognition-The development and vision of the three-generation mobile learing[J].China Educational Technology,2007,(6):7-18

[2] Xu Guangyou,Shi Yuanchun, Xie Weikai.Pervasion computing[J].Computer Journal,2003,(9):1042-1050

[3] Li Jiahou.From courseware are to intergrableeare. The new delevopment of CAI in Chinese school classes[J].China Education Technology[J].1997,(4):12-16

[4] Siemens G.Connectivism:a learing theory for the digital age[J].Instuctional Technology \& Distance Learning.2005,(1):3-9

[5] Tim Berners-Lee,James Hendler.Ora Lassila.The Semantic Web[J].Scientific American,2001,284(5):34-43 